\title{
ITERATIVE WINDOWED PARAMETER ESTIMATION OF MULTIPLE SUPERIMPOSED DAMPED EXPONENTIALS IN NOISE
}

\author{
Shanglin Ye and Elias Aboutanios \\ School of Electrical Engineering and Telecommunications \\ The University of New South Wales, Sydney, Australia \\ Email: shanglin.ye@unsw.edu.au, elias@ieee.org
}

\begin{abstract}
The research problem of the parameter estimation of multiple superimposed damped complex exponentials in noise is of significant importance in many engineering and science applications. In this paper, we propose a simple yet accurate estimator to address the problem. By combining an efficient windowed frequency and damping estimator for a single component with an iterative leakage subtraction scheme, the novel method consecutively and iteratively estimates one component at a time by gradually reducing the leakage introduced by other components presented. Simulation results are presented to verify that the proposed algorithm is capable of outperforming state-of-art time and frequency domain algorithms.
\end{abstract}

Index Terms - Parameter estimator, damped exponential, interpolation algorithm, nuclear magnetic resonance spectroscopy.

\section{INTRODUCTION}

We address the parameter estimation problem of the following signal model

$$
x(n)=\sum_{i=1}^{I} A_{i} e^{\left(-\eta_{i}+j 2 \pi f_{i}\right) n}+w(n), \quad n=0 \ldots N-1,
$$

where $N$ is the total number of signal samples. $I$ is the number of components and is assumed to be known a priori. $A_{i}, f_{i} \in$ $[-0.5,0.5]$ and $\eta_{i}>0$ are respectively the complex amplitude, the frequency and the damping factor of the $i^{\text {th }}$ component, which we aim to estimate. $w(n)$ are the additive Gaussian noise terms with zero mean and variance $\sigma^{2}$.

(1) is considered to be the ideal signal model in many applications including low frequency mechanical spectroscopy [1] and nuclear magnetic resonance spectroscopy [2]. Thus, estimating the parameters of the signal has always been of significant interest. Various parameter estimation algorithms have been proposed to solve this problem [3], among which the time-domain high resolution parametric estimators, such as $[2,4]$, are the most popular ones. They are based on the singular value decomposition (SVD) to separate the noisy signal into pure signal and noise subspaces. They can achieve accurate estimation while are also capable of resolving closely separated components. However, the main drawback of these algorithms comes to the high computational cost for performing the SVD operation and Hankel matrix inversion, which has a complexity of $O\left(N^{3}\right)$. The frequency-domain estimators based on interpolation on Fourier coefficients, such as those presented in $[5,6,7,8]$ and [9], on the other hand, are computationally more efficient. But they obtain biased estimates when applied to the multiple component case. The estimation bias can be reduced using methods in [10] and [11], where pre-windowing the signal before interpolation is performed, however with a trade-off of increased estimation variance. To overcome these limitations, in this paper, we put forward a novel efficient algorithm that operates in the frequency domain and at the same time can achieve accurate parameter estimation.

The rest of the paper is organised as follows. In Section 2, we present the novel parameter estimation algorithm. In Section 3, we demonstrate the simulation results of the proposed algorithm. Finally, conclusion is drawn in Section 4.

\section{THE PROPOSED METHOD}

The proposed method utilise the generalised Iterative Windowed A\&M (IWAM) estimator to estimate the frequency and damping factor of each component in combination with a leakage subtraction scheme proposed in [12]. We start the section by presenting the generalised IWAM estimator.

\subsection{The Generalised IWAM Estimator}

From now on, we denote $\hat{\lambda}$ as the estimate of $\lambda$. The generalised IWAM estimator, extended from the original IWAM estimator presented in [5], is capable of achieving accurate estimation of the frequency and damping factor of an arbitrarily zero-padded damped exponential in additive Gaussian noise.

Assuming $x(n)$ to be a single damped exponential in the presence of noise and $K=p N(p \in \mathbb{Z})$ to be the zero-padded 
signal length. The generalised IWAM estimator starts by finding the maximum bin of the $K$-point periodogram of the signal as the coarse estimation of the frequency

$$
\hat{m}=\arg \max _{k}|X(k)|^{2},
$$

where $X(k)=K-\operatorname{DFT}[x(n)]$. The true frequency is then given by

$$
f=\frac{\hat{m}+\delta}{K},
$$

where $\delta \in[-0.5,0.5]$ is the frequency residual. The coarse estimation is followed by the fine estimation which finds $\delta$ and $\eta$ based on iterative interpolation on Fourier coefficients. Before each iteration, the signal is windowed by an exponential decaying function $e^{-\gamma n}$ and the previous estimate of the frequency residual $\hat{\delta}$ is removed from the maximum bin. For zero-padded signals where $p>1$, according to related results shown in $[13,14]$, the optimal locations of interpolation are dependent on $p$ and are at locations $\left(\hat{m}+\hat{\delta} \pm \frac{p}{2}\right)$ of the signal spectrum. The noise-free coefficients interpolated at the optimal locations in each iteration are computed by

$$
\begin{aligned}
X_{ \pm \frac{p}{2}} & =\sum_{n=0}^{N-1} x(n) e^{-\gamma n-j \frac{2 \pi}{K}\left(\hat{m}+\hat{\delta} \pm \frac{p}{2}\right) n} \\
& =A \frac{1+e^{-N(\eta+\gamma)+j \frac{2 \pi}{p}(\delta-\hat{\delta})}}{1-e^{-(\eta+\gamma)+j \frac{2 \pi}{K}\left(\delta-\hat{\delta} \mp \frac{p}{2}\right)}} .
\end{aligned}
$$

Let

$$
z=e^{-(\eta+\gamma)+\frac{2 \pi}{K}(\delta-\hat{\delta})},
$$

and following similar derivation presented in [13], the estimator of $z$ is calculated as

$$
\hat{z}=\frac{X_{\frac{p}{2}}-X_{-\frac{p}{2}}}{e^{-\frac{j \pi}{N}} X_{\frac{p}{2}}-e^{\frac{j \pi}{N}} X_{-\frac{p}{2}}},
$$

and the estimates of $\delta$ and $\eta$ in each iteration are given by

$$
\hat{\delta}=\frac{K}{2 \pi} \angle \hat{z}+\hat{\delta}, \quad \text { and } \quad \hat{\eta}=-\ln |\hat{z}|-\gamma
$$

Same as what has been presented in [5], to obtain the minimum variance that approaches the Cramer-Rao Lower Bound (CRLB), the algorithm is run for two iterations. In the first iteration, $\gamma$ and $\hat{\delta}$ are set to zero. In the second iteration, $\gamma$ should be chosen based on the equality $\gamma=r \hat{\eta}$ where $\hat{\eta}$ is the estimate of damping factor from the first iteration. The ratio $r$ is given by

$$
r= \begin{cases}0.02 N^{2} \hat{\eta}^{2}+0.02 N \hat{\eta}+0.39 & 0<N \hat{\eta} \leq 3 \\ 1-1.08 e^{-0.41 N \hat{\eta}}-0.07 e^{-0.08 N \hat{\eta}} & N \hat{\eta}>3\end{cases}
$$

The estimation procedure of the generalised IWAM estimator is summarised in Table 1.
Table 1. The Generalised IWAM estimator

\begin{tabular}{ll}
\hline \hline 1. & Set $K=p N, p=1,2, \ldots ;$ \\
2. & Calculate $X(k)=K-\mathrm{FFT}[x(n)] ;$ \\
3. & Find $\hat{m}=\arg \max _{k}|X(k)|^{2} ;$ \\
4. & Initialise $\hat{\delta}=\hat{\eta}=\gamma=0 ;$ \\
5. Loop the following steps for two iterations \\
(1) Calculate $X_{ \pm p / 2}$ by (4); \\
(2) Calculate $\hat{z}$ by (6); \\
(3) Renew $\hat{\delta}$ and $\hat{\eta}$ by (7); \\
(4) Calculate $\tau=N \hat{\eta}$, find $r$ by (8) and \\
renew $\gamma=r \hat{\eta} ;$ \\
\hline \hline
\end{tabular}

\subsection{The Multi-tone Estimator}

Now we extend the generalised IWAM estimator to the multitone $(I \geq 2)$ case. Let $\hat{m}_{i}(i=1, \ldots, I)$ be the estimated maximum bins of the components and are assumed in this work to be identical to the true values. The frequencies are now

$$
f_{i}=\frac{\hat{m}_{i}+\delta_{i}}{K}, \quad i=1 \ldots I .
$$

The exponential windows being applied are $e^{-\gamma_{i} n}, i=1 \ldots I$ where $\gamma_{i}$ can vary from component to component. The windowed noise-free interpolated Fourier coefficients of the $i^{\text {th }}$ component in each iteration are

$$
\tilde{X}_{i, \pm \frac{p}{2}}=X_{i, \pm \frac{p}{2}}+\sum_{l=1, l \neq i}^{I} \breve{X}_{l, \pm \frac{p}{2}}
$$

where $X_{i, \pm \frac{p}{2}}$ are the expected coefficients for a single exponential as shown in (4). $\breve{X}_{l, \pm \frac{p}{2}}(l=1 \ldots I, l \neq i)$, on the other hand, are the leakage terms introduced by the other $I-1$ existing components, which can be calculated by

$$
\begin{aligned}
\hat{\ddot{X}}_{l, \pm \frac{p}{2}} & =\sum_{n=0}^{N-1} x_{l}(n) e^{-\gamma_{i} n-j \frac{2 \pi}{K}\left(\hat{m}_{i}+\hat{\delta}_{i} \pm \frac{p}{2}\right) n} \\
& =A_{l} \frac{1+e^{-N\left(\eta_{l}+\gamma_{i}\right)+j \frac{2 \pi}{p} \Delta_{l}}}{1-e^{-\left(\eta_{l}+\gamma_{i}\right)+j \frac{2 \pi}{K}\left(\Delta_{l} \mp \frac{p}{2}\right)}}
\end{aligned}
$$

where $\Delta_{l}=\left(\hat{m}_{l}-\hat{m}_{i}\right)+\left(\delta_{l}-\hat{\delta}_{i}\right)$. Therefore, the reduction of the estimation error can be performed by subtracting the sum of leakage from the interpolated coefficients to obtain the estimates of the expected coefficients of a single exponential. Substituting (11) into (10) yields

$$
\hat{X}_{i, \pm \frac{p}{2}}=\tilde{X}_{i, \pm \frac{p}{2}}-\sum_{l=1, l \neq i}^{I} A_{l} \frac{1+e^{-N\left(\eta_{l}+\gamma_{i}\right)+j \frac{2 \pi}{p} \Delta_{l}}}{1-e^{-\left(\eta_{l}+\gamma_{i}\right)+j \frac{2 \pi}{K}\left(\Delta_{l} \mp \frac{p}{2}\right)}} .
$$

From the expression of (12) we find the true values of $\delta_{l}$, $\eta_{l}, A_{l}(l=1 \ldots I, l \neq i)$ are unknown and should also be estimated during the estimation process. In [12], this is solved 


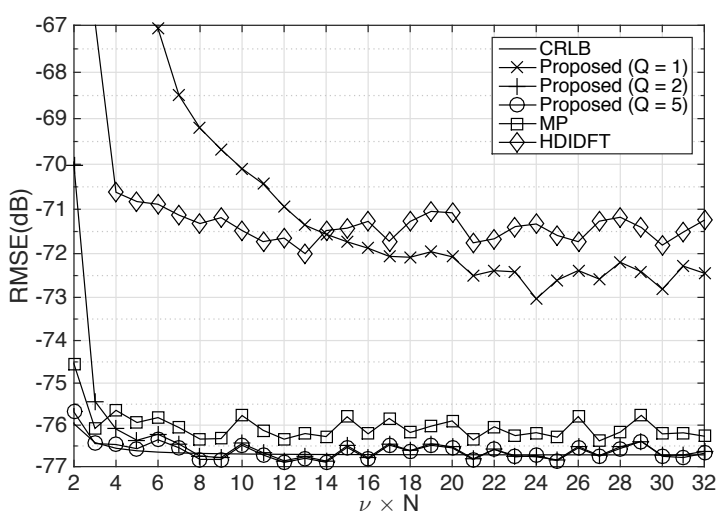

Fig. 1. RMSE of $\hat{f}_{1}$ versus frequency intervals $v N$ when SNR $=30 \mathrm{~dB}, N=64$ and $\eta_{1}=\eta_{2}=0.02$. 1,000 Monte Carlo runs were used.

by iteratively estimating all the parameters of one component at a time with leakage subtraction incorporated. During the process, the sum of the leakage terms in (12) are constructed by the parameter estimates of all the other $I-1$ components obtained in the previous iteration. As the leakage subtraction process runs iteratively, it enables the reduction of the error between $\hat{X}_{i, \pm \frac{p}{2}}$ and $X_{i, \pm \frac{p}{2}}$. The estimation of $\delta_{i}$ and $\eta_{i}$ can be obtained using (7) by substituting (12) into (6), while the estimation of $A_{i}$ can be obtained by

$$
\hat{A}_{i}=\frac{\sum_{n=0}^{N-1} x(n) e^{\left(-\hat{\eta}_{i}-j 2 \pi \hat{f}_{i}\right) n}-\sum_{l \neq i} \hat{X}_{l, \hat{f}_{i}}}{\sum_{n=0}^{N-1} e^{-2 \hat{\eta}_{i} n}}
$$

where

$$
\hat{\grave{X}}_{l, \hat{f}_{i}}=\hat{A}_{l} \frac{1-e^{-N\left(\hat{\eta}_{l}+\hat{\eta}_{i}\right)+j 2 \pi N\left(\hat{f}_{l}-\hat{f}_{i}\right)}}{1-e^{-\left(\hat{\eta}_{l}+\hat{\eta}_{i}\right)+j 2 \pi\left(\hat{f}_{l}-\hat{f}_{i}\right)}}
$$

is the leakage term at frequency $f_{i}$ introduced by the $l^{\text {th }}$ component. The procedure of the proposed algorithm is finally summarised in Table 2.

Table 2. The Multi-tone IWAM Estimator

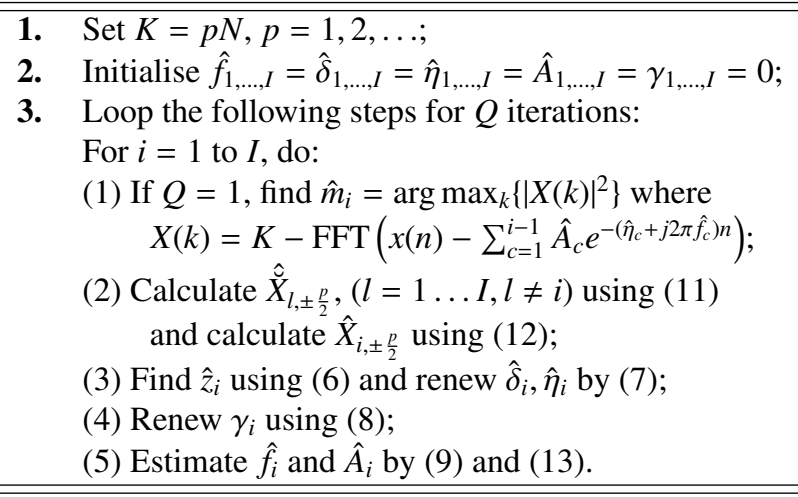

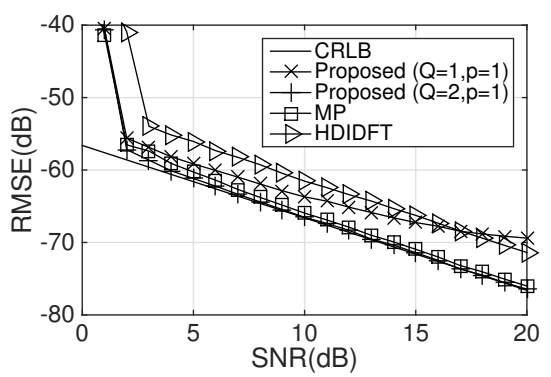

(a) $\hat{f}_{1}$

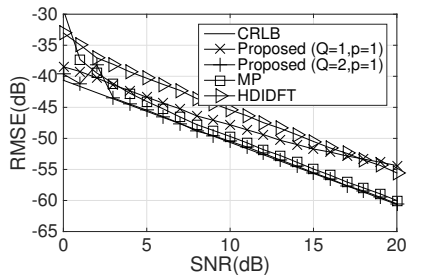

(b) $\hat{\eta}_{1}$

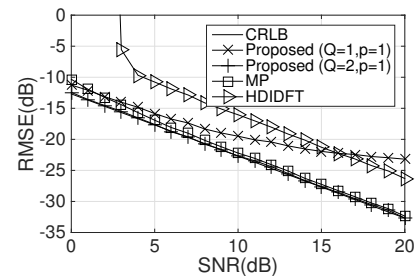

(c) $\left|\hat{A}_{1}\right|$
Fig. 2. RMSE of estimates of the first component in (15) versus SNR when $v=0.1, N=64$ and $\eta_{1}=\eta_{2}=0.02 .5,000$ Monte Carlo runs are used.

\section{SIMULATION RESULTS}

In this section, we present the simulation results of the proposed multi-tone estimator and verify its performance. We test the algorithm on the following signal

$$
x(n)=\left|A_{1}\right| e^{j \phi_{1}} e^{-\eta_{1} n+j 2 \pi f_{1} n}+\left|A_{2}\right| e^{j \phi_{2}} e^{-\eta_{2} n+j 2 \pi\left(f_{1}+v\right) n}+w(n),
$$

where $n=0 \ldots N-1 . v$ is the interval between the two frequencies. $\left|A_{1}\right|$ and $\left|A_{2}\right|$ are fixed to 1 and $\phi_{1}$ is set to zero. $f_{1}$ and $\phi_{2}$ are randomly selected in $[-0.5,0.5-v]$ and $[-\pi, \pi]$ respectively in each Monte Carlo run. We define the (nominal) signal to noise ratio (SNR) [15] as $\rho=1 / \sigma^{2}$.

First we investigate the performance of the algorithm versus frequency separation $v$. In this test we set $\eta_{1}=\eta_{2}=0.02$, $\mathrm{SNR}=30 \mathrm{~dB}$ and $N=64$. We vary $v N$, which is the difference of the maximum bins between the two components, from 2 to $N / 2=32$. For the sake of benchmarking the performance, the novel method is compared with the CRLB [16], the time domain method Matrix Pencil (MP) [4] with the pencil parameter always set to $L=\lfloor N / 3\rfloor$ and the frequency domain windowing method Hann Damped Interpolated DFT (HDIDFT) [11]. The proposed algorithm in this test is implemented using $p=1$ and $Q=1,2$ and 5. In Fig. 1 we show the root mean square error (RMSE) of $\hat{f}_{1}$ versus $v N$. The results of the other parameters are similar and not shown here due to the page limit. We can see that when $Q \geq 5$, the RMSE is extremely close to the CRLB as $v N \geq 5$. As the number of iterations $Q$ increases, we are able to obtain lower RMSE for 


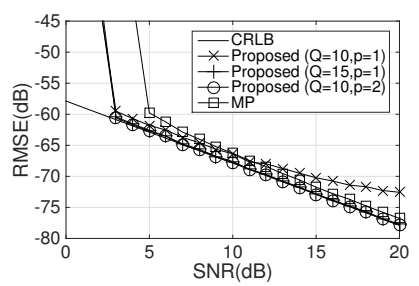

(a) $\hat{f}_{1}$

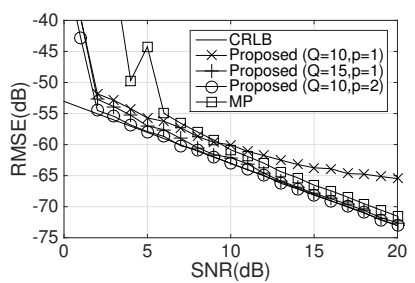

(c) $\hat{\eta}_{1}$

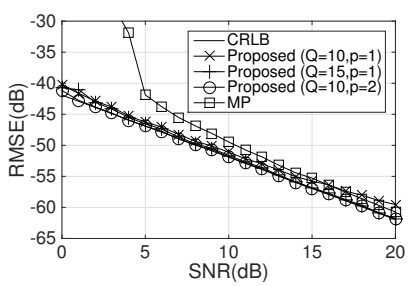

(b) $\hat{f}_{2}$

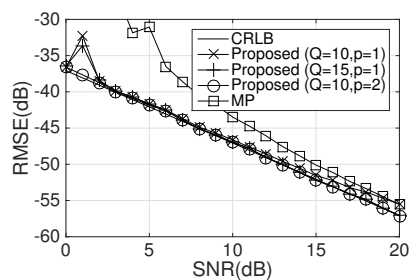

(d) $\hat{\eta}_{2}$
Fig. 3. RMSE of estimates of frequencies and damping factors in (15) versus SNR when $v=0.02, N=64$ and $\eta_{2}=10 \eta_{1}=0.02$. 5,000 Monte Carlo runs are used.

small $v N$, where the frequencies are closely separated. Notice that the proposed method is capable of obtaining RMSE that is marginally smaller than MP for all $v N$, while the HDIDFT gives unreliable results when $v N<4$ and has RMSE that are approximately $6 \mathrm{~dB}$ higher than CRLB when $v N \geq 4$.

Then we examine the RMSE of the parameter estimates versus SNR. Fig. 2 shows the RMSE of $\hat{f}_{1}, \hat{\eta}_{1}$ and $\left|\hat{A}_{1}\right|$ versus SNR when $v=0.1, N=64$ and $\eta_{1}=\eta_{2}=0.02$. The proposed algorithm is implemented using $p=1$ and $Q=1,2$. We only show the related results of the first component as those of the second component are similar. We can find that for $\mathrm{SNR} \geq 3 \mathrm{~dB}$, two iterations are enough for the proposed algorithm to outperform both MP and HDIDFT by achieving CRLB-comparable performance. In Fig. 3 we show the RMSE of $\hat{f}_{1}, \hat{\eta}_{1}, \hat{f}_{2}$ and $\hat{\eta}_{2}$ obtained by various methods versus SNR when another set of signal parameters, $v=0.02$ and $\eta_{2}=10 \eta_{1}=0.02$, is used. In this test the proposed algorithm is implemented using $p=1,2$ and $Q=10,15$. As demonstrated in Fig. 1, when frequencies are close to each other, the HDIDFT is not be able to obtain comparable results with the proposed method and MP. So the results of HDIDFT are not shown in this test. We can find from the figures that for the proposed algorithm, when $p=1$, the estimation bias is reduced as the iteration number increases from $Q=10$ to $Q=15$, and there is no observable bias at $20 \mathrm{~dB}$ when $Q=15$. On the other hand, the RMSE follows the CRLB by requiring less number of iteration when $p=2$, because coefficients closer to the true maxima are used as the signal is zero-padded. It is also clear from the figures that the proposed algorithm can achieve smaller RMSE than MP at all SNR, especially having a lower breakdown threshold.

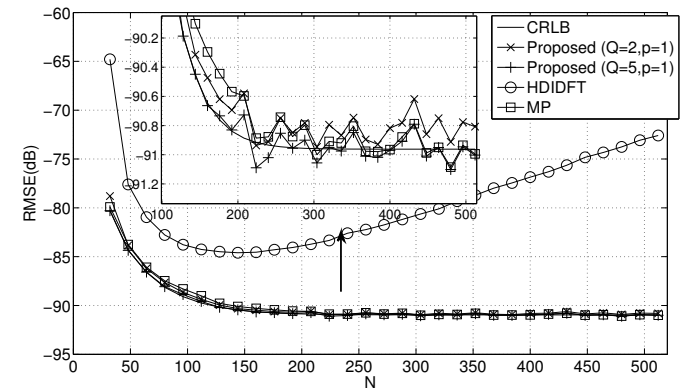

(a) $\hat{f}_{1}$

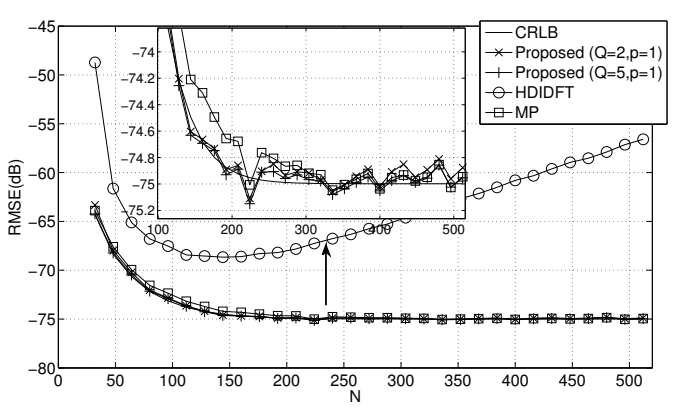

(b) $\hat{\eta}_{1}$

Fig. 4. RMSE of $\hat{f}_{1}$ and $\hat{\eta}_{1}$ versus $N$ when $\mathrm{SNR}=30 \mathrm{~dB}$, $v=0.1$ and $\eta_{1}=\eta_{2}=0.02$. 5,000 Monte Carlo runs were used.

We finally look at the performance of the novel estimator versus the number of samples $N$. We set $\eta_{1}=\eta_{2}=0.02$, $\mathrm{SNR}=30 \mathrm{~dB}$ and $v=0.1 . p=1$ and $Q=2,5$ are used in the proposed estimator. Fig. 4 shows the RMSE of $\hat{f}_{1}$ and $\hat{\eta}_{1}$ versus $N$, which varies from 32 to 512. The results of the other parameters, which exhibit similar behaviour, are not shown here due to the lack of space. We find that when $Q=5$, the proposed method approaches the CRLB and beat HDIDFT for all $N$. Also, it attains observable lower RMSE than MP when $N<300$.

Before the end of this section, it is worth pointing out that the computational complexity of the proposed algorithm has the same order as that of the FFT operation, $O\left(N \log _{2} N\right)$, which is more efficient than the SVD based high resolution methods, which require $O\left(N^{3}\right)$ for computation.

\section{CONCLUSION}

We presented in this paper a novel method for estimating the frequencies, damping factors and complex amplitudes of arbitrarily zero-padded multiple superimposed damped complex exponentials in additive Gaussian noise. The proposed algorithm iteratively performs the generalised Iterative Windowed A\&M (IWAM) algorithm to estimate the parameters of one 
single component at a time in combination with a leakage subtraction scheme. During the iterative process, the leakage of the interpolated Fourier coefficients introduced by other components is gradually subtracted and the error between the interpolated coefficients and their expected values of the single component case is therefore reduced. Simulation results demonstrated that the proposed algorithm can achieve estimation variance that is extremely close to the CRLB.

\section{REFERENCES}

[1] K. Duda, L.B. Magalas, M. Majewski, and T.P. Zielinski, "DFT-based estimation of damped oscillation parameters in low-frequency mechanical spectroscopy," IEEE Transactions on Instrumentation and Measurement, vol. 60, no. 11 , pp. $3608-3618,2011$.

[2] S. Vanhuffel, H. Chen, C. Decanniere, and P. Vanhecke, "Algorithm for time-domain NMR data fitting based on total least squares," Journal of Magnetic Resonance, Series A, vol. 110, no. 2, pp. 228 - 237, 1994.

[3] T.P. Zielinski and K. Duda, "Frequency and damping estimation methods - an overview," Metrology and Measurement Systems, vol. 18, no. 4, pp. 505-528, 2011.

[4] Y. Hua and T. K. Sarkar, "Matrix pencil method for estimating parameters of exponentially damped/undamped sinusoids in noise," IEEE Transactions on Acoustics, Speech, and Signal Processing, vol. 38, no. 5, pp. 814824, 1990.

[5] E. Aboutanios and S. Ye, "Efficient iterative estimation of the parameters of a damped complex exponential in noise," IEEE Signal Processing Letters, vol. 21, no. 8, pp. 975-979, 2014.

[6] M. Bertocco, C. Offelli, and D. Petri, "Analysis of damped sinusoidal signals via a frequency-domain interpolation algorithm," IEEE Transactions on Instrumentation and Measurement, vol. 43, no. 2, pp. 245-250, 1994.

[7] R.-C. Wu and C.-T. Chiang, "Analysis of the exponential signal by the interpolated DFT algorithm," IEEE Transactions on Instrumentation and Measurement, vol. 59, no. 12, pp. 3306-3317, Dec 2010.

[8] B.G. Quinn, "Estimating frequency by interpolation using Fourier coefficients," IEEE Transactions on Signal Processing, vol. 42, no. 5, pp. 1264-1268, May 1994.

[9] E. Jacobsen and P. Kootsookos, "Fast, accurate frequency estimators [DSP Tips Tricks]," IEEE Signal Processing Magazine, vol. 24, no. 3, pp. 123-125, May 2007.
[10] K. Duda and S. Barczentewicz, "Interpolated DFT for $\sin ^{\alpha}(x)$ windows," IEEE Transactions on Instrumentation and Measurement, vol. 63, no. 4, pp. 754-760, 2014.

[11] R. Diao and Q. Meng, “An interpolation algorithm for discrete Fourier transforms of weighted damped sinusoidal signals," IEEE Transactions on Instrumentation and Measurement, vol. 63, no. 6, pp. 1505-1513, 2014.

[12] S. Ye and E. Aboutanios, "An algorithm for the parameter estimation of multiple superimposed exponentials in noise," in The 40th International Conference on Acoustic, Speech and Signal Processing (ICASSP 2015), 2015.

[13] S. Ye and E. Aboutanios, "Efficient 2-D frequency and damping estimation by interpolation on Fourier coefficients,” IEEE Signal Processing Letters, vol. 20, no. 2, pp. 137-140, 2013.

[14] E. Aboutanios, "Generalised DFT-based estimators of the frequency of a complex exponential in noise," in Proceedings - 2010 3rd International Congress on Image and Signal Processing, 2010, vol. 6, pp. 2998-3002.

[15] E. Aboutanios, "Estimation of the frequency and decay factor of a decaying exponential in noise," IEEE Transactions on Signal Processing, vol. 58, no. 2, pp. 501-509, 2010.

[16] F. Qian, S. Leung, Y. Zhu, W. Wong, D. Pao, and W. Lau, "Damped sinusoidal signals parameter estimation in frequency domain," Signal Processing, vol. 92, no. 2 , pp. $381-391,2012$. 